\title{
Channel Assignment based on Routing Decisions (CARD): Traffic-Dependent Topology Control for Multi-Channel Networks
}

\author{
Ryan E. Irwin and Luiz A. DaSilva \\ Wireless@Virginia Tech \\ Bradley Department of Electrical and Computer Engineering \\ Virginia Polytechnic Institute and State University \\ email: \{reirwin, ldasilva\}@vt.edu
}

\begin{abstract}
Dynamic spectrum access (DSA) holds the promise for more efficient utilization of the spectrum, while requiring greater cooperation between PHY, MAC, and NET layers to allocate resources and dynamically react to changing network conditions. In this paper, we propose Channel Assignment based on Routing Decisions (CARD), a mechanism that combines channel assignment and topology control so that at any given time the cognitive network self-organizes into the topology that is best suited to support the current offered traffic. CARD is a distributed mechanism, and each network node relies on local information only. We show that CARD results in an improvement in route length (and, thus, end-to-end delay), aggregate network capacity, and, in some situations, energy efficiency.
\end{abstract}

\section{INTRODUCTION}

Dynamic spectrum access (DSA) is a proposed idea for better utilizing the spectrum [1]. However, many issues stand in the way of an actual network implementation. Currently, most of the efforts have been placed in issues related to the physical layer for DSA. Although these issues, including spectrum sensing and signal classification, are not yet resolved, work on higher layers is imperative to the progress of DSA networks, and may ease the requirements on the physical layer.

Specifically, when channel assignment decisions are made by the PHY/MAC layers, such assignments combined with the setting of transmit power, induce a certain network topology. Routes are then determined over this existing topology.

An alternative approach is to consider end-to-end and network goals when making channel selection decisions, according to the concept of cognitive networks articulated in [2]. Instead of the topology dictating which routes are feasible, information about active network flows can be used to assign channels and thus induce a topology that is desirable from the point of view of the network.

This work is partially supported by a Bradley Fellowship and by DARPA through Air Force Research Laboratory (AFRL) Contract FA8750-07-C-0169. The views and conclusions contained in this document are those of the authors and should not be interpreted as representing the official policies, either expressed or implied, of the Defense Advanced Research Projects Agency or the U.S.Government.
In the process of designing a DSA medium access control (MAC) scheme, new abstractions that span the physical, data-link, and network layers must be developed. This design encompasses the traditional PHY/MAC/NET functions along with new issues such as neighbor discovery in a multi-channel environment, rendezvous, and intelligent channel assignment.

In this paper, we propose an approach that combines channel assignment and topology control, so that at any given time the network self-organizes into the topology that is best suited to support the current offered traffic. This approach, Channel Assignment based on Routing Decisions (CARD), is a distributed mechanism where each node makes decisions given its local information. Each node inspects the current route and attempts to shorten the route through dynamic selection of channels. It produces, whenever feasible, both an interference-free channel assignment for nodes actively involved in routing and shorter routes from source to destination. Preliminary results indicate that this method of jointly assigning channels and routes has benefits such as reduced delay and increased network capacity. In some cases energy-efficiency improvements are also obtained, and if enough channels are available intra- and inter-flow interference avoidance techniques can be applied to improve network throughput.

The contribution of this paper is two-fold:

1) Building on work on multi-channel MAC protocols and topology control algorithms, we define a design framework for a MAC scheme that is tailored to the needs of DSA;

2) We propose a channel assignment mechanism that takes into account end-to-end data delivery goals and provide an evaluation of potential benefits of this approach.

This paper is organized as follows. Section II outlines previous work in multi-channel MAC protocols and topology control. A PHY/MAC/NET framework is proposed in Section III. In Section IV the CARD algorithm is explained. Section V provides results from simulations of the distributed implementation of the algorithm. Section VI offers some concluding thoughts and outlines future work on this topic. 
Table I. Summary of Multi-channel Protocols (see text for references).

\begin{tabular}{|c|c|c|c|c|c|c|c|}
\hline Current Protocols & Neighbor Discovery* & Data Exchange Setup* & Synch & T'cvers & Sensing* & Ctrl Ch & Dynamic Ch Sets* \\
\hline Multi-Channel MAC(McMAC or MMAC) & Blind, Accidental & Hop to receiver \& RTS/CTS & Mild & 1 & No & 0 & No \\
\hline Cognitive MAC (C-MAC) & Blind & Schedule NAV & High & 1 & Yes & 1 & Yes \\
\hline Dedicated Ctrl Channel (DCA, MTMA) & Assumed $(1 \mathrm{ctrl} \mathrm{ch})$ & RTS/CTS on ctrl channel & None & 2 & No & 1 & No \\
\hline Common Hopping (CHMA, HRMA \& RICHDP) & Assumed & RTS/CTS on current hop & Mild & 1 & No & 0 & No \\
\hline Slotted Seeded Channel Hopping (SSCH) & Assumed & Wait for hop & Mild & 1 & No & 0 & No \\
\hline Split Phase (MAP) & Assumed $(1 \mathrm{ctrl} \mathrm{ch})$ & Schedule NAV & Mild & 1 & No & 1 & No \\
\hline On-Demand Channel Switching (ODC) & Mildly Assumed & RTS/CTS (multi ch attempts) & None & 1 & No & 0 & No \\
\hline
\end{tabular}

* indicates a requirement of DSA

\section{RELATED WORK}

It is helpful to investigate current multi-channel MAC protocols, qualitatively assessing their strengths and weaknesses. We briefly discuss the extension of these protocols to provide for the requirements of DSA. Also, a concise overview of TC algorithms is presented.

\section{A. Multi-Channel MAC Protocols}

We classify multi-channel MAC protocols in the literature as: protocols using a common control channel; protocols relying on hopping patterns; and protocols implementing on-demand switching (see Table I for a summary).

In one approach, a dedicated control channel is used to set up transfers on one of several data channels. For examples refer to [3] - [6]. The use of a common control channel simplifies rendezvous. A major drawback is that the control channel can become congested or unavailable due to a primary user and possibly lead to underutilization of the data channels. Another drawback is that either a transceiver is dedicated for control purposes for at least a portion of a duty cycle.

Common hopping protocols rely on devices hopping along with others in a common hopping pattern to communicate. Examples include [7] - [9]. A common hopping pattern precludes the need for a common control channel. A disadvantage is that synchronization is necessary. Achieving synchronization is not trivial, yet assumed in many MAC protocol designs. Also, a channel switching penalty means additional overhead.

Instead of having a community hopping pattern, devices can have their own hopping sequence. Examples include [10] and [11]. The improvement over having a common hopping pattern is that multiple rendezvous can happen simultaneously on orthogonal channels [12], but devices must obtain neighboring hopping patterns. Since switching channels costs a device time and power, the On-Demand Channel switching (ODC) protocol [13] provides a way to utilize multiple channels without synchronization, but may make rendezvous more difficult as the list of available channels grows.

\section{B. Topology Control Algorithms}

A TC algorithm focused on energy efficiency can eliminate all of the links except for the minimum energy cost links that maintain network connectivity. Examples include [14] and [15], where minimum spanning trees (MSTs) result. The advantage of MSTs is that they result in low network overhead from minimal MAC- and NET-layer messaging. The biggest deficiency is they result in a limited set of links that flows have to utilize, so some links can become congested. Furthermore, if any link is removed, as might occur in a DSA environment, the network becomes disconnected.

Another energy-efficiency approach seeks to minimize the physical length of links as in a Gabriel graph. In [16], a subset of the Gabriel tree is examined. The advantages are clear, but when energy is not a major concern it may make more sense to utilize longer distance links.

Another approach is to seek high spectral efficiency, specifically, limiting the topology to using the fewest number of channels necessary. The reader is referred to [17] for a comparison of TC algorithms with some optimizing power- and spectralefficiency. Also, [17] proposes a game-theoretic approach to power control and then channel assignment.

Separate from power and spectral optimizations, [18] evaluates a scheme forming links based on the queue sizes of the nodes forming the link. The idea is to give priority to assigning links and channels based on the need for each link. Note that this paper is not to be confused with [19] which outlines an algorithm, coincidentally called CARD, which makes channel decisions using factors including queue size.

\section{FRAMEWORK}

To develop a MAC for a multi-channel DSA environment, new PHY/MAC/NET cross-layer interactions may be defined to enable more intelligent channel assignments. This leads to developing new abstractions to more clearly define components of the lower layers.

The architecture proposed in Fig. 1 is employed in the remainder of the paper. It is designed to be as flexible as possible to fit a variety of future DSA component implementations. A brief walk-through is provided for the block-diagram. The bold pathways are heavily utilized paths by the proposed CARD algorithm described in the next section.

The network layer maintains its own view of the network topology. If a flow-based channel assignment protocol is used (as proposed), the channel assignment algorithm will be performed in this block.

The Frame Manager is responsible for the encapsulation of packets into frames at the transmitter and the decapsulation at the 


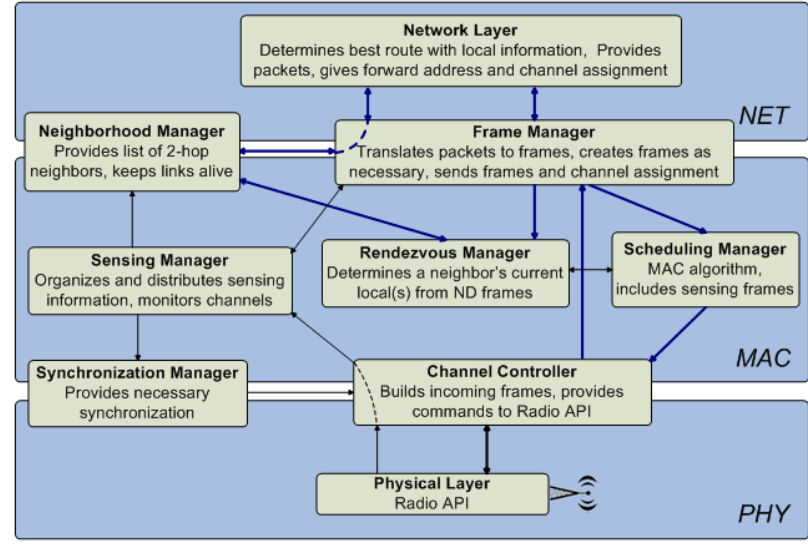

Fig. 1. A modular architecture for PHY/MAC/NET layer functionality.

receiver. It sends outgoing frames along with control information to the Scheduling Manager for transmission.

A list of one- and two-hop neighbors is maintained by the Neighborhood Manager. The list includes channel occupancy and other statistics such as the number of transceivers. A Rendezvous Manager handles dynamic channel occupancies by harvesting neighbor discovery (ND) information.

The Scheduling Manager block receives frames and channel information from the Frame Manager. It can query neighboring channel occupations. If multiple transceivers are used multiple queues could reside in this block.

Primary user detection is the job of the Sensing Manager. Requests for sensing frames are generated by this block. Much work has focused on DSA sensing and classification. Different levels of synchronization may be necessary and are provided by the Synchronization Manager.

The Channel Controller interacts with the physical layer's transceiver(s) through commands handling I/O of frames. The actual transmission and reception of signals occurs at the physical layer, which may support multiple transceivers.

\section{Channel Assignment Algorithm}

We now introduce some notation adopted to describe the algorithm. Denote $G\left(N, E_{A}\right)$ as the network, where $N$ represents the set of nodes and $E_{A}$ the set of all active edges. Each node is assumed to have built a view of the topology from traditional NET layer messaging to form this graph. Denote $R_{T}$ as each radio's transmit range. Edge $e_{i j}$ exists if nodes $i$ and $j$ are within range, $R_{T}$, of each other. Define $E$ as the set of all possible edges, so $E_{A} \subset E$. Denote $M_{i}$ as the set of channels available at node $i$ and $M_{i j}$ as $M_{i} \cap M_{j}$. Let $e_{i j}^{m}$ be the active edge between nodes $i$ and $j$ on channel $m$. If necessary the algorithm updates $m$ to ensure its eligibility in the set $M_{i j}$ resulting in an adaption of an existing link to avoid intra- and inter-flow interference. Denote $N_{i}^{(1)}$ and $N_{i}^{(2)}$ as the potential one- and two-hop sets of neighbors of node $i$ respectively. Define $R_{p q}$ as the route from node $p$ to node $q$ such that the ordered vector $\left(n_{1}, n_{2}, n_{3}, \ldots, n_{r}\right)$ is formed where $n_{1}=n_{p}$ and $n_{r}=n_{q}$.

The inputs to node $i$ given a packet to forward are $G\left(N, E_{A}\right)$, $N_{i}^{(1)}, N_{i}^{(2)}$, and $M_{j} \forall j \in N_{i}^{(1)} \cup N_{i}^{(2)}$. Also, the default route

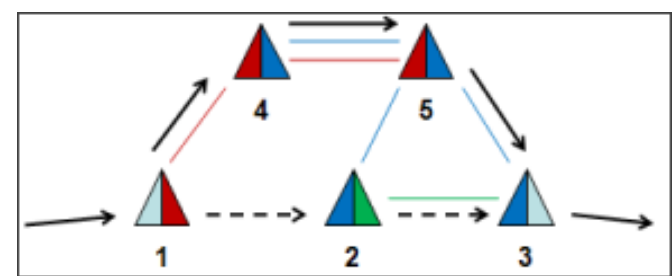

Fig. 2. Example of a two-hop adaptation shortening a flow's route

$R_{i d}$, where $d$ is the destination, is known to $i$. The output is an activation of a potential link to use for forwarding to shorten the route if possible. If no shorter route is found forwarding is performed according to the forwarding table. If enough channels are available, forwarding occurs on a non-interfering channel to eliminate inter- and intra-flow interferences.

The main idea of the algorithm is for the node to look at the expected route vector and scroll through each index starting with the destination looking to see if any hop is in $N_{i}^{(1)} \cup N_{i}^{(2)}$. The algorithm looks to shorten the route by bridging the gap between itself and a node further along the route.

Label node of reference $i=1$ and the destination index $d=$ $r$ on the route vector. Subroutine Forge_Link $\left(n_{i}, n_{j}, m_{i j}\right)$ calls for edge $e_{i j}^{m}$ to be forged between nodes $i$ and $j$ on channel $m$. The Forward_Packet $\left(n_{j}\right)$ subroutine schedules a transmission the link to reach neighbor $n_{j}$. It is assumed that all matrices $M_{i j}$ are updated dynamically through neighbor discovery beacon messages when nodes broadcast available and in-use channels. Subroutine Forward_Packet $\left(n_{j}\right)$ will adapt the link (if possible) to be non-interfering with any surrounding links.

Distributed CARD Algorithm

(1) for $n_{i}$ in $R_{1 r}$ where $i$ decrements from $r$ downto 3

(15) Forward_Packet $\left(r_{2}\right)$

(16) exit algorithm

The main loop will run at most the length of a route, upperbounded by the network diameter. The worst case network diameter is $n$, but a more practical network (as simulated) is upper-bounded by $O(\sqrt{n})$. It is assumed that all nodes maintain their potential neighbor tables in an ordered array (e.g. based on id), so searches are on order of $O(\log n$ ) (if not $O(n)$ for a linear search). The overall complexity of CARD in a lattice topology is $O(\sqrt{n} \log n)$. Note that calculating every neighbor's 


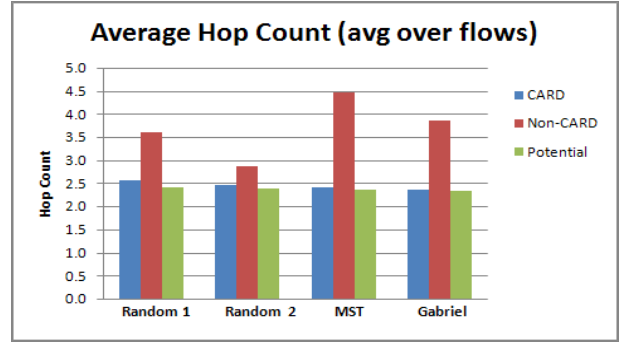

Fig. 3. Hop count reduction.

$M_{i j}$ matrix introduces a complexity of $O(m n)$, with $m$ being the number of channels, but this can be maintained dynamically.

Figure 2 shows an example of the distributed CARD algorithm performed at Node 1. Each triangle represents a node, and different shaded halves of the triangle represent different transceivers and their channel occupancy. Node 1 has to make a forwarding decision. The solid arrows indicate the expected path to reach Node 3 (enroute to the destination). Node 1 discovers that through appropriate channel selection, it can reach Node 3 in two hops instead of the present three, so it forges a link and forwards the packet to Node 2 indicated by the dashed arrows. Advantages of this approach: it is fully distributed, has low complexity, and guarantees a performance improvement assuming constant link rates.

\section{RESUlTS}

This section describes our simulation results. We assess the benefits of CARD in terms of reduced route length (hop count), increased aggregate capacity of the network and, in some cases, reduced energy consumption per flow.

\section{A. Simulation}

Simulation of the distributed algorithm was performed in Java. At the start of the simulation a topology is generated. There were four types of initial topologies built upon a $7 \times 7$ lattice of nodes prior to injecting network traffic.

1) Random 1 Topology: When nodes are deciding which channel to occupy, they negotiate with one neighbor (at random) to occupy one available channel. This simulates a rendezvous process. Note if other neighboring nodes choose like channels, links are formed. (The average node degree is approximately 3.5 for 15 channels.)

2) Random 2 Topology: Nodes pick channels in a similar fashion as the Random 1 topology, except nodes negotiate with two neighbors. (The average node degree is approximately 5 for 15 channels.)

3) Minimum Spanning Tree: We choose one of the multiple MSTs that can be found for the lattice topology.

4) Gabriel Tree: A full Gabriel tree was used. A full tree serves as a best case scenario for energy consumption since the shortest links are used.

When packets reach the destination we extract route length information; the experiment is repeated with and without CARD. Also, a theoretical minimum potential route length is calculated.

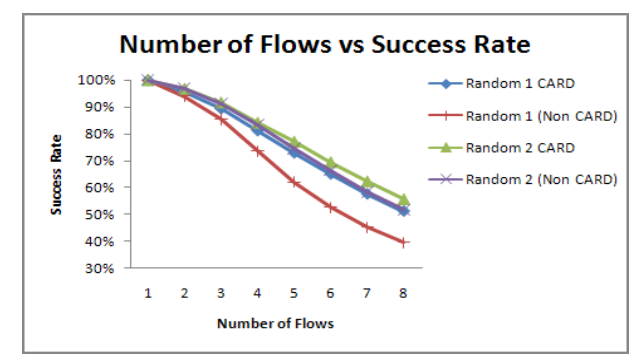

Fig. 4. Random topologies flow support.

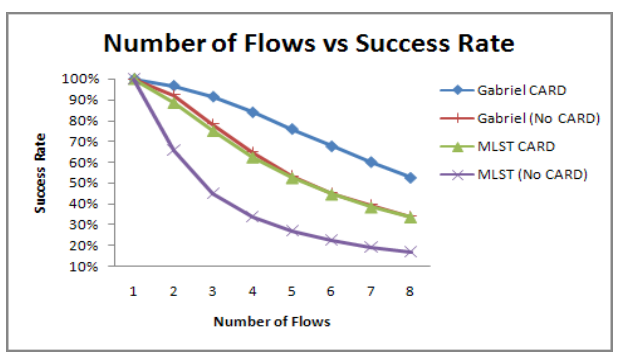

Fig. 5. Optimized topologies flow support.

To achieve this minimum, every potential edge must be known network-wide (unlike CARD, which acts on local information). Energy calculations are reported to compare the amount of energy required to service a flow under different topology control/channel assignment schemes.

\section{B. Average Hop Count Reduction}

Three measurements are provided per topology illustrating the effectiveness of CARD at acheiving its primary goal of reducing hop count. The plots are labeled as previously described with the plot labeled potential being a measure of the theoretical minimum hop count where all nodes have global ND (i.e. nodes perform traditional routing based on knowledge of all possible links, $E$, and all $M_{i j}$ sets).

Figure 3 shows that the hop counts were reduced on all tested topologies. The reduction of hop count varied little as more flows were introduced, so an average was taken from all measurements from 1 to 8 network flows. Results indicate that using only local ND information was sufficient to acheive on average within $3 \%$ of the theoretical minimum hop count.

\section{Average Flow Count Support}

When the CARD algorithm is adopted, shorter routes are used for servicing a flow whenever possible. This means that there is an increase in the number of nodes available to carry other flows. The result is that the number of flows that can be simultaneously supported by the network will increase, i.e. the network capacity is increased.

This particular implementation illustrates the capability of CARD when taken as a snapshot in time at any given instant, so the number of flows a node is allowed to participate in is limited to one flow. The idea is that if a simple time sharing approach is used, the number of flows supported would scale up. Overall, each network was compared on the same terms, for fairness 


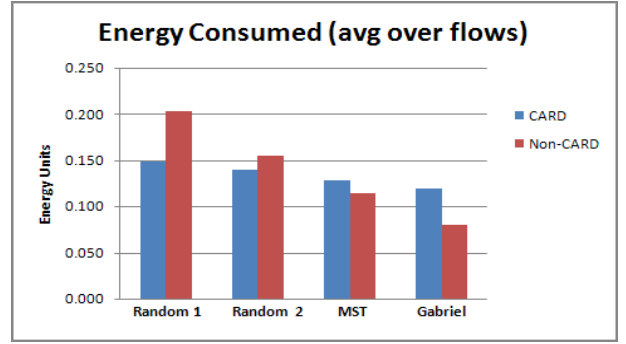

Fig. 6. Random topologies energy consumption.

in comparisons. Figures 4 and 5 show an improvement in the number of flows supported for all topologies tested. The capacity improvement per topology is proportional to its improvement in hop count.

\section{Energy Efficiency}

A tradeoff in energy consumption exists between having fewer forwarding transmissions and utilizing longer, higher energy, links. Figure 6 illustrates the impact on energy used in the network when flows are successfully delivered in CARD and non-CARD networks. In random topologies, there is a small improvement in energy consumed, but CARD increased energy consumption when employed on the already energy-optimized MLST and Gabriel network topologies.

\section{CONCLUSions ANd Future WorK}

Traditionally, channel assignment has been handled primarily in the PHY/MAC layers. However, the NET layer has the privilege of having a broader network view, so this can be exploited to provide more intelligent channel assignment for the network.

The Channel Assignment based on Routing Decisions (CARD) algorithm demonstrates the benefits of channel assignment influenced by the network layer in an ad hoc network. These benefits include:

1) A flow's end-to-end delay can be reduced due to using a shorter route.

2) Network capacity can be increased because more flows can be supported.

3) Network energy consumption is reduced in some situations as a result of fewer forwarding transmissions.

4) Although not evaluated in this study, throughput can be improved when enough channels are available by avoiding intra- and inter-flow interferences.

The next challenge is to incorporate CARD in a network simulator (e.g. ns-2, OMNet). Use in a network simulator will more realistically model the full network protocol stack, and various mobility models can be used for testing.

Investigation is necessary for identifying scenarios where it does not make sense to employ CARD. Scenarios could include a one-packet flow or a situation where one node changes its channel assignment(s) and disconnects the network.

The NET layer can also leverage knowledge of neighboring queue sizes yielding a possible enhancement of the algorithm to handle heavily congested links by incorporating back-pressure techniques [20]. This would allow CARD to exploit multiple routes while forwarding, balancing the network's load.

\section{REFERENCES}

[1] Q. Zhao and B. M. Sadler, "A Survey of Dynamic Spectrum Access," IEEE Signal Processing Magazine, vol. 24, no. 3, pp. 79-89, 2007.

[2] R. W. Thomas, D. H. Friend, L. A. DaSilva, and A. B. MacKenzie, "Cognitive Networks: Adaptation and Learning to Achieve End-to-end Performance Objectives," IEEE Communications Magazine, vol. 44, no. 12, pp. 51-57, 2006.

[3] S. Wu, C. Lin, Y. Tseng, J. Sheu, "A New Multi-Channel MAC Protocol with On-Demand Channel Assignment for Multi-Hop Mobile Ad Hoc Networks,' Proc. IEEE International Symposium on Parallel Architectures, Algorithms and Networks (I-SPAN), pp. 232-237, 2000.

[4] C. Xu, G. Li, W. Cheng, Z. Yang, "Multi-Transceiver Multiple Access (MTMA) for Mobile Wireless Ad Hoc Networks," Proc. IEEE International Conference on Communications, pp. 2932-2936, 2005.

[5] J. Chen, S. Sheu, and C. Yang, "A New Multichannel Access Protocol for IEEE 802.11 Ad Hoc Wireless LANs," Proc. IEEE International Symposium on Personal, Indoor and Mobile Radio Communications, 2003.

[6] C. Cordeiro, K. Challapali, "C-MAC: A Cognitive MAC Protocol for Multi-Channel Wireless," Proc. IEEE Dynamic Spectrum Access Networks Conference, pp. 145-157, 2007.

[7] Z. Tang, J.J. Garcia-Luna-Aceves, "Hop Reservation Multiple Access (HRMA) for Multichannel Packet Radio Networks," Proc. 7th IEEE International Conference on Computer Communications and Networks, pp. 388-395, 1998.

[8] A. Tzamaloukas, J.J. Garcia-Luna-Aceves, "Channel-Hopping Multiple Access," Proc. IEEE International Conference on Communications, pp. 415 419, 2000.

[9] A. Tzamaloukas, J.J. Garcia-Luna-Aceves, "A Receiver-initiated Collisionavoidance Protocol for Multi-channel Networks," Proc. 20th Annual Joint Conference of the IEEE Computer and Communications 0 (INFOCOM), pp. 189-198, 2001

[10] H.W. So, J. Walrand, and J. Mo, "McMAC: A Parallel Rendezvous Multi-Channel MAC Protocol," Proc. IEEE Wireless Communications and Networking Conference, pp. 334-339, 2007.

[11] P. Bahl, R. Chandra, and J. Dunagan, "SSCH: Slotted Seeded Channel Hopping for Capacity Improvement in IEEE 802.11 Ad Hoc Wireless Networks," Proc. ACM MobiCom, pp. 216-230, 2004.

[12] J. Mo, H. W. So, J. Walrand, "Comparison of Multichannel MAC Protocols," IEEE Transactions on Mobile Computing, pp. 50-65, 2008.

[13] P. Porwal and M. Papadopouli, "On-Demand Channel Switching for MultiChannel Wireless MAC Protocols," Technical Report TR04-024, University of North Carolina at Chapel Hill, 2004.

[14] X.-L. Li, K. Moaveni-Nejad, W.-Z. Song, and W.-Z. Wang, "Interferenceaware topology control for wireless sensor networks," Proc. of IEEE SECON 2005, pp. 263-274, 2005.

[15] K.-D. Wu and W. Liao, "On Constructing Low Interference Topology in Multihop Wireless Networks," Proc. of IEEE ICC 2006, 2006.

[16] T. Johansson and L. Carr-Motyčková, "Reducing interference in ad hoc networks through topology control,' Proc. of 2005 Joint Work on Found. of Mob. Comp., pp. 17-23, 2005.

[17] R.W. Thomas, R.S Komali, A.B. MacKenzie, L.A. DaSilva, "Joint Power and Channel Minimization in Topology Control: A Cognitive Network Approach," Proc. IEEE International Conference on Communications, pp. 6538-6543, 2007.

[18] X. Lin, S. Rasool, "A Distributed Joint Channel-Assignment, Scheduling and Routing Algorithm for Multi-Channel Ad-hoc Wireless Networks," Proc. IEEE International Conference on Communications, pp 1118-1126, 2007.

[19] C. Ghosh, D. P. Argrawal, "Channel Assignment with Route Discovery (CARD) using Cognitive Radio in Multi-channel Multi-radio Wireless Mesh Networks," Proc. IEEE Workshop on Networking Technologies for Software Defined Radio Networks, pp. 36-41, 2006.

[20] M.J. Neely, E. Modiano, C.E. Rohrs, "Dynamic Power Allocation and Routing for Time Varying Wireless Networks," IEEE Journal on Selected Areas in Communication, vol. 23, no. 1, pp. 89-103, 2005. 